\title{
Temperature gradient snow metamorphosis
}

\author{
SIGNE KJELSTRUP RATKJE
}

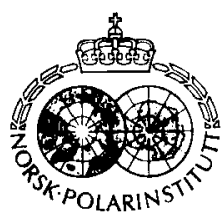

Ratkje, S. K. 1985: Temperature gradient snow metamorphosis. Polar Research 3 n.s., 141-143.

This note shows how the rate of ice crystal growth during temperature gradient metamorphosis can be calculated using irreversible thermodynamics. The method describes the transfer of mass as a consequence of heat transfer. The main heat transfer is the latent heat change by condensing and freezing. Local equilibrium and no convection is assumed. The transport equation, with the temperature gradient as the force of transport, predicts mass transfer measurements.

Signe Kjelstrup Ratkje, Laboratory of Physical Chemistry, Norwegian Institute of Technology, N-7034, Trondheim, Norway; October 1984 (revised March 1985).

Snow metamorphosis takes place under constant temperature conditions or under exposure to temperature gradients (Perla 1978; Colbeck 1982). When snow metamorphosis takes place in sloping terrain, strength against shear stress is lowered and avalanches may occur (Haefeli 1939). This is one reason why the formation of snow metamorphosis should be described quantitatively.

The equi-temperature metamorphism can be described by water transport due to differences in surface energy (Perla 1978). This note concerns the metamorphosis due to temperature gradients only, neglecting the effect of shapes of the crystals.

The fact that ice crystals grow in snow exposed to strong vertical temperature gradients (Akitaya 1974) indicates that heat transport is somehow coupled to mass transport. This means that mass transport should not be described by simple transport laws like Fick's or Darcy's law. These equations contain only one driving force and no interference or coupling terms. One way to include the coupling between the fluxes in the equations is to apply irreversible thermodynamics. It is the purpose of this article to do so, in order to bring out the coupling effect and provide equations for this transport. For a general reference text on irreversible thermodynamics, Fitts (1962) may be referred to.

The possibility of thermal convection as a cause of mass transfer has been discussed by Palm \& Tveitereid (1979). Thermal convection can be predicted for large gradients and old snow with big pores. This case is not included in the present investigation, since we are dealing with the condition of local equilibrium and no convection.
Local equilibrium means that the water vapour in the pores of the snow is saturated, and that equilibrium thermodynamics can be applied to each volume element even if transport takes place across it. Since the temperature varies along the pore, the vapour pressure will do the same. However, it will be shown that this pressure variation is not the primary force of transport. The thermal force obtained from irreversible thermodynamics explains mass transfer by diffusion.

\section{The coupled transport of heat and mass}

The general equations for coupled transport of heat and mass are from irreversible thermodynamics (see e.g. Førland \& Ratkje 1981):

$J_{q^{\prime}}=-L_{11} \nabla \ln T-L_{12} \nabla \mu_{w T}$

$J_{w}=-L_{21} \nabla \ln T-L_{22} \nabla \mu_{w} T$

$J_{q^{\prime}}$ is the measurable total heat flux across the region of transport, including latent heat changes. $J_{w}$ is the total mass flux of the chemical component $\mathrm{H}_{2} \mathrm{O}$. This can be followed by measuring crystal growth rates and/or snow density changes. The gradient in chemical potential of $\mathrm{H}_{2} \mathrm{O}$ at constant temperature is $\nabla \mu_{w T}$. This term consists generally of two parts at absolute temperature $T$ :

$\nabla \mu_{w T}=\nabla \mu_{w}^{c}+V_{w} \nabla P$

The reference chosen for $\mu_{w}$ is the enthalpy of ice in the bottom of the snow layer. Since local equilibrium exists, we may consider either the liquid phase or the vapour phase in order to find 
$\nabla \mu_{w T}$. The concentration dependent part, $\nabla \mu_{w}^{c}$, is zero for a pure component. The second part of $\nabla \mu_{w}$ contains the pressure gradient $\nabla P$ and the partial molar volume of water. Since $\nabla P$ is zero for the liquid phase, this term is also zero. There is a pressure gradient for the vapour phase, but this is due to temperature variation, and $\nabla \mu_{w T}$ in equations $(1+2)$ does not include the temperature effect.

Our objective is to give an equation for the mass flux. For the thermodynamic condition $\nabla \mu_{w}=0$, the result from equation (2) is

$J_{w}=-L_{21} \nabla \ln T$

The next problem is to interpret the coefficient $L_{21}$. The coefficients $L_{i j}$ are generalized transport coefficients which obtain their meaning through defined experiments. Since Onsagers relations are valid:

$L_{12}=L_{21}$

we may choose to determine $L_{12}$. In order to find $L_{12}$, consider first the case of no temperature gradients. Heat may then be transferred only if water is transferred and gives off latent heat by condensing and/or freezing. The ratio between equations (1) and (2) gives:

$\left(\frac{J_{q^{\prime}}}{J_{w}}\right)_{\Delta T=0}=\frac{L_{12}}{L_{22}}$

Since $\Delta T=0$, this ratio gives the reversible heat transferred per mole of water. This ratio should be close to the total enthalpy change by the phase transition(s), denoted $\Delta H$ from now

$\frac{L_{12}}{L_{22}}=\Delta H$

We are thus able to find $L_{12}$ from equations $(6,7)$ by determining $L_{22}$. The coefficient $L_{22}$ can most easily be determined when $\nabla T=0$ and $\nabla P=0$. From equations (2) and (3) we have

$J_{w}=-L_{22} \nabla \mu_{w}^{c}=-L_{22} R T \frac{\nabla C}{C}$

Here $C$ is the vapour concentration and $R$ is the gas constant. Equation (8) describes the mass flux as due to molecular diffusion. Molecular diffusion is ordinarily described by Fick's law, when the temperature is constant:

$J_{w}=-D \nabla C$

By comparing equations (8) and (9) we find $L_{22}$ from the diffusion constant $D$
$L_{22}=D \frac{C}{R T}$

The information obtained through equations (5), (7) and (10) can now be used in equation (4) to give

$J_{w}=-\frac{D C}{R T} \frac{\Delta H}{T} \nabla T$

Since for a phase transition $\Delta H / T=\Delta S$, where $\Delta S$ is the entropy change, we may rewrite equation (11) to

$J_{2}=-\frac{D C}{R T} \Delta S \nabla T$

This equation shows that there will be a continuous transfer of water and thus snow metamorphosis as long as temperature gradients are maintained. The temperature gradient is the primary force of transport and the proportionality coefficient $D$ is dependent on the mechanism of mass transfer. In the development of equation (12) this mechanism was chosen to be diffusion in the vapour phase. Water may also be transferred on the grain surface, see e.g. Colbeck (1980).

The following characteristic values are taken from Palm \& Tveitereid (1979) and Perla (1978).

$$
\begin{aligned}
& D=2.2 \cdot 10^{-5} \mathrm{~m}^{2} \mathrm{~s}^{-1} \\
& C=2 \cdot 10^{-3} \mathrm{~kg} \mathrm{~m}^{-3} \\
& \Delta T=-10 \mathrm{Km}^{-1} \\
& T=263 \mathrm{~K}
\end{aligned}
$$

The gas constant is equal to $R=8.31 \mathrm{JK}^{-1} \mathrm{~mol}^{-1}$ and the entropy of sublimation for ice is $\Delta S=$ $145 \mathrm{JK}^{-1} \mathrm{~mol}^{-1}$ from standard thermodynamic tables. This gives $J_{w}=3 \cdot 10^{-8} \mathrm{~kg} \mathrm{~m}^{-2} \mathrm{~s}^{-1}$ from equation (12).

\section{Discussion and conclusion}

Trabant \& Benson (1972) refer to an experiment where large, thin polyethylene plastic sheets were installed at $10 \mathrm{~cm}$ vertical intervals in the snow. The snow was exposed to temperature gradients, and the formation of depth hoar was observed between the layers. Vertical vapour transport was halted between the layers. In this experiment convection in the snow is minimized, and mass transfer occurs due to diffusion. Trabant \& Benson observed a mass transfer rate about $10^{-7} \mathrm{~kg} \mathrm{~m}^{-2} \mathrm{~s}^{-1}$. This value may be compared to 
the one calculated here $\left(3 \cdot 10^{.8} \mathrm{~kg} \mathrm{~m}^{-2} \mathrm{~s}^{-1}\right)$, since the experimental conditions should be contained in the development of equation (12). The difference between the two values is probably not greater than what should be expected from experimental uncertainties.

Perla (1978) has also presented a theoretical estimate of mass transfer rates for gradients about $10 \mathrm{Km}^{-1}$, giving the same order of magnitude as our value. The theoretical base for our transport equation is, however, different from this analysis. The main difference is that the present analysis uses the temperature gradient as a direct driving force of transport, while traditionally Fick's law of diffusion has been used as the basic equation. The temperature gradient snow metamorphosis is in some respects similar to the formation of ice lenses during frost heave: the same transport equations can be used for both (Førland \& Ratkje 1981).

The present theoretical equations can be applied to either of the predominant mechanisms of mass transfer, diffusion in the vapour phase, or transport on the grain surface (Colbeck 1982).

The present analysis cannot be used to distinguish between the mechanisms of transfer. The result of the analysis shows, however, how thermal diffusion may explain snow metamorphosis due to temperature gradients.

\section{References}

Akitaya, E. 1974: Some experiments on the growth of depth hoar. Physics of snow and ice, pt. 2. Proc. Sapporo Conf. 1966 (Hokkaido Univ.), Inst. Low Temp. Sci., 713-723.

Colbeck, S. C. 1980: Thermodynamics of snow metamorphosism due to variations in curvature. J. of Glaciology 26, 291.

Colbeck, S. C. 1982: An overview of seasonal snow metamorphosism. Rev. Geophys. and Space Physics 20, 45.

Fitts, D. 1962: Nonequilibrium Thermodynamics. McGrawHill, New York.

Førland, T. \& Ratkje, S. K. 1981: Irreversible thermodynamic treatment of frost heave. Engineering Geol. 18, 225.

Haefeli, R. 1939: Schneemechanik mit Hinweisen auf die Erdbaumechanik. In Bader, H., Haefeli, R., Bucher, E., Neher, J., Eckel, O. \& Thams, Chr. (eds.): Der Schnee und Seine Metamorphose. Beitrage zur Geologie der Schweiz, Geotechnische Serie, Hydrologie, Lieferung 3. Bern.

Palm, E. \& Tveitereid, M. 1979: On heat and mass flux through dry snow. J. Geophys. Res. 84, 745.

Perla, R. 1978: Temperature gradient and equi-temperature metamorphosism. Deuxieme Rencontre Internationale sur la Neige et les Avalanches, Grenoble, France. 43 pp.

Trabant, D. \& Benson, C. 1972: Field experiments on the development of depth hoar. Geol. Soc. Am. Mem. 135, 309. 